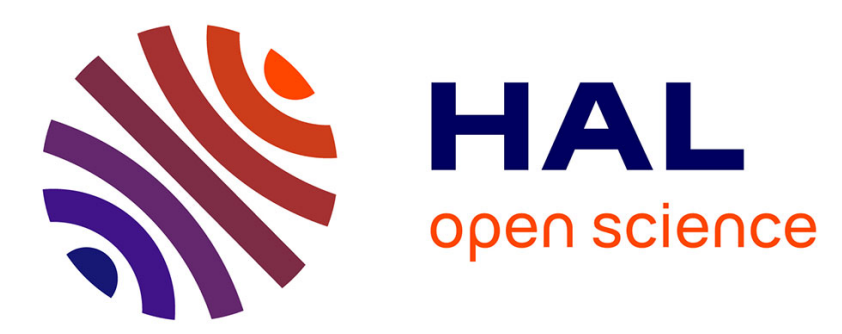

\title{
Réforme agraire et reconfiguration du régime de gouvernementalité rurale dans Les Tuxtlas, Mexique, 1920-1945
}

Eric Léonard

\section{- To cite this version:}

Eric Léonard. Réforme agraire et reconfiguration du régime de gouvernementalité rurale dans Les Tuxtlas, Mexique, 1920-1945. Critique Internationale, 2017, La gouvernementalité rurale dans les pays du Sud, 75, pp.53-69. 10.3917/crii.075.0053 . ird-01532187

\section{HAL Id: ird-01532187 \\ https://hal.ird.fr/ird-01532187}

Submitted on 2 Jun 2017

HAL is a multi-disciplinary open access archive for the deposit and dissemination of scientific research documents, whether they are published or not. The documents may come from teaching and research institutions in France or abroad, or from public or private research centers.
L'archive ouverte pluridisciplinaire HAL, est destinée au dépôt et à la diffusion de documents scientifiques de niveau recherche, publiés ou non, émanant des établissements d'enseignement et de recherche français ou étrangers, des laboratoires publics ou privés. 


\title{
Réforme agraire et reconfiguration du régime de gouvernementalité rurale
}

\author{
dans Les Tuxtlas, Mexique, 1920-1945
}

\author{
Eric Léonard*
}

\section{Introduction}

Depuis une quinzaine d'années, la situation sociale et politique de grandes régions du Mexique rural a été marquée par une forte instabilité combinant deux phénomènes corrélés. Cette instabilité résulte en premier lieu d'une violence diffuse mais généralisée, ponctuée de phases d'intensification lors des processus électoraux, moments où s'expriment les logiques de contrôle territorial des différents cartels, via la cooptation des candidats, dans un contexte de décentralisation qui a accru les compétences et les ressources des gouvernements locaux. L'accroissement de la compétition électorale et de ses formes violentes n'est pas sans lien avec le délitement d'un régime de gouvernementalité rurale qui reposait sur les régulations corporatives assurées par deux types d'institutions complémentaires : les premières, territoriales, ont été mises en place dans le cadre de la réforme agraire, avec la création de nouvelles communautés, les ejidos, auxquelles étaient assignés un territoire en propriété collective et des instances de gouvernement concentrant des fonctions étendues de régulation (foncière, politique, productive); les secondes, socioprofessionnelles, correspondent aux médiations assumées par une fédération syndicale unique, intégrée au parti officiel et chargée de construire les compromis au sein du secteur paysan et de négocier et d'arbitrer la désignation de ses représentants aux différents niveaux de gouvernance. La fonctionnalité des ces régulations explique dans une large mesure la longévité du régime politique postrévolutionnaire au Mexique et la relative stabilité du secteur rural, dans un continent agité par la violence rurale au long du $\mathrm{XX}^{\mathrm{e}}$ siècle ${ }^{1}$.

La construction de ce régime de gouvernementalité est indissociable de la mise en œuvre d'une réforme agraire qui s'est étendue, au long d'un demi-siècle, à la moitié de la surface nationale, a conduit à la création de plus de 30000 ejidos et a concerné trois générations successives. La réforme agraire a été la culmination d'une entreprise de construction nationale et étatique qui a fait de la politique foncière un de ses leviers fondamentaux. Pour les libéraux qui assumèrent le pouvoir durant la seconde moitié du XIX ${ }^{e}$ siècle, le démantèlement des propriétés collectives des communautés et municipalités et l'imposition d'un régime de propriété privée étaient les conditions de la diffusion d'un modèle de citoyenneté individuelle qui s'imposerait aux formes de représentation collective et unanimiste ayant cours dans les sociétés indiennes et qui forcerait leur intégration à un projet unifié de Nation. Le résultat de cette politique, menée dans un contexte d'incorporation brutale aux marchés, a été une concentration foncière sans précédent ${ }^{2}$ et la montée de tensions sociales qui débouchèrent sur la révolution des années 1910-1919. Les gouvernements issus de ce mouvement

\footnotetext{
* IRD, UMR GRED et Laboratoire Mixte International MESO, eric.leonard@ird.fr

${ }^{1}$ Au cours du long règne du Parti Révolutionaire Institutionnel (PRI), entre 1929 et 2000, des soulèvements violents ont secoué certaines zones rurales mexicaines, mais ils ont été contrôlés par la combinaison d'actions ciblées de répression et de cooptation des mécontentements, précisément à travers les instances de régulation corporatives que le régime avait mises en place. L'incapacité de ce dernier à juguler le soulèvement zapatiste de 1994, au Chiapas, a révélé la perte de fonctionnalité de ces instances, en même temps qu'elle a marqué le déclin définitif du régime postrévolutionnaire.

2 En 1905, 0,2\% des familles rurales (2,5\% des proriétaires) détenaient $87 \%$ de la surface agricole du pays, alors que $91 \%$ d'entere elles n'avaient pas de terre (Sector Agrario, La transformación agraria. Origen, evolución, retos, Mexico, Secretaria de Reforma Agraria, 1997: 30-31).
} 
ont inscrit au rang de devoir constitutionnel la satisfaction des revendications fondamentales des groupes paysans qui s'étaient mobilisés autour du slogan Tierra y Libertad : d'une part, la restitution des terres spoliées aux communautés et aux famille rurales et, d'autre part, la restauration de l'autonomie politique des municipalités que le régime libéral avait placé sous la tutelle de l'Etat.

La combinaison de ces deux revendications portait en germe le risque du rétablissement de communautés alliant les pouvoirs de régulation foncière et de représentation politique collective, dans un contexte de restauration précaire de l'Etat. La constitution de 1917 ne définissant pas les prérogatives juridiques des trois niveaux de gouvernement légal (Etat central, gouvernements provinciaux, municipalités) dans la conduite des procédures de redistribution des terres, la mise en œuvre de la réforme agraire est devenue un enjeu central de reconstruction du régime politique et de redéfinition des rapports de gouvernance.

Le présent article propose d'éclairer les enjeux de renégociation du régime de gouvernementalité rurale dans le contexte contemporain de décentralisation politique et de violence, à partir de l'examen des processus de mise en œuvre de la réforme agraire et de leur incidences sur les rapports entre l'Etat, les municipalités et les différentes composantes des communautés rurales dans une région indienne située sur le littoral du Golfe du Mexique. Il montre comment la réforme agraire, comme toute proposition de réforme institutionnelle, a été saisie par certains secteurs de la société locale (élites municipales, propriétaires terriens, jeunes soumis aux structures d'autorité patriarcales, villages marginalisés dans les hiérarchies politiques) pour altérer en leur faveur les rapports de pouvoir. Nous verrons en particulier comment la politique foncière a été mobilisée comme ressource par des secteurs subalternes de cette société pour alimenter des processus de frontière interne conduisant à la construction de nouveaux territoires et de nouveaux corps politiques, et comment la violence a constitué une dimension centrale de ces processus.

\section{La première phase de la réforme agraire et le projet de restauration d'une "communauté municipale"}

\section{Le "commun municipal" et la fusion des sphères de régulation agraire et politique autour de la municipalité}

Les débats et mobilisations autour de la réforme agraire à San Andrés Tuxtla ont été profondément marqués par l'histoire récente du démantèlement du "commun municipal", à la fin du XIX siècle, et des accaparements fonciers dont il fut l'occasion ${ }^{3}$. Le statut des terrains communaux avait constitué en enjeu récurrent de conflits pour le contrôle de l'institution municipale. Ceux-ci avaient été acquis en 1839 par une poignée de grands commerçants aux héritiers de la famille Cortés, qui les avait reçus en fief en récompenses des services rendus lors de la conquête du Mexique. Outre l'usage personnel qu'en faisaient ces grands commerçants, ces terrains étaient la principale source de financement de la municipalité via, les fermages perçus auprès de ses ressortissants. Bien qu'ils fussent couramment évoquées comme formant le "commun municipal", ils furent gérés pendant une grande partie du $\mathrm{XIX}$ siècle par une Société Agricole, formée de 74 membres liés aux grandes familles commerçantes qui les avaient acquis ${ }^{4}$. Le contrôle de ces terres, dont dépendait la vie des quelques 5000 familles agricoles résidant dans la municipalité, a assuré aux membres de la Société Agricole la mainmise sur le pouvoir municipal, les mandats de préfet et d'administrateur fiscal, ou encore de commandant de la Garde Nationale dans le canton des Tuxtlas, jusqu'aux années 1880.

À cette époque, les recompositions des marchés agricoles liées à l'incorporation du Mexique dans les échanges internationaux ont été directement à l'origine de l'affaiblissement du groupe des grands

\footnotetext{
3 Voir Eric Léonard, “Marché foncier, asymétries de pouvoir et exclusion. Incidences régionales des réformes libérales mexicaines de la fin du XIXe siècle", Economie Rurale, 2008, 303-305: 136-153.

${ }^{4}$ Sur le commun de San Andrés Tuxtla, voir Eric Léonard, Dynamique du changement légal et construction territoriale dans les Tuxtlas (Mexique), Habilitation à diriger des recherches, Paris, Univ. Paris 1 Panthéon-Sorbonne, 2014, T. III, chap. 4.
} 
commerçants et de la perte de fonctionnalité du système de patronage qu'ils avaient mis en place autour de la "municipalité agraire" pour organiser l'accès aux ressources foncières, économiques et politiques. L'éviction de ce groupe du pouvoir local a précipité le démantèlement du commun municipal et sa répartition en lopins privés, en 1886. L'issue de cette politique a été un processus accéléré de dépossession des petits bénéficiaires à travers les opérations de crédit mises en place par des maisons de négoce international et leur représentants locaux, et la construction de très grandes propriétés ${ }^{5}: 25$ ans après le fractionnement du "commun", le nombre de propriétaires avait diminué de $85 \%$ (de 3750 à 585) et 11 personnes contrôlaient $70 \%$ de la surface répartie en 1886. Au regard de ces mécanismes de spoliation, la gestion des terres par la Société Agricole et la municipalité a été associée dans l'imaginaire populaire à un "âge d'or" de l'agriculture paysanne qui a fortement pesé dans les processus ultérieurs de positionnement vis-à-vis des lois de réforme agraire

\section{La résurgence de la municipalité comme acteur politique autonome et la tentative de reconstitution du commun municipal}

La polarisation des structures agraires dans la région ne semble pas y avoir constitué un levier de mobilisation sociale lors du conflit révolutionnaire. Des milices paysannes y ont opéré, mais en tant que composantes de groupes armés plus larges, dont les champs d'action se situaient plus au sud, près des axes de communication et des grandes plantations de canne à sucre, qui étaient les principaux enjeux de confrontation ${ }^{6}$. Entre 1913 et 1920, les représentants de l'ancien ordre politique ont conservé le contrôle de la municipalité, avec la bienveillance du gouvernement provincial de Veracruz. Une contestation radicale a toutefois émergé à partir de 1916, autour de la formation du Parti Rouge de San Andrés, qui regroupait des figures de l'armée révolutionnaire et des guérillas paysannes, ainsi que des membres de l'aile agrariste du Parti libéral. En 1920, mettant à profit la rébellion du général Obregón contre le président Carranza, les radicaux du Parti Rouge, alliés aux milices zapatistes implantées au sud de la région, prirent d'assaut la place de San Andrés et destituèrent le conseil municipal. Lors des élections générales qui suivirent la prise du pouvoir par Obregón, le Parti Rouge remporta l'ensemble des mandats, alors que le colonel Adalberto Tejeda était élu gouverneur du Veracruz. Au long de la décennie suivante, le Parti Rouge de San Andrés a intégré le complexe politique et militaire formé par Tejeda pour imposer un projet de transformation des structures politiques, dont le mode de gouvernance s'appuyait sur l'intégration fonctionnelle des milices paysannes, des conseils municipaux et du gouvernement provincial et dont le mode de régulation reposait sur la gestion de la réforme agraire.

Jusqu'alors, les redistributions de terre avaient eu une faible incidence dans l'Etat de Veracruz. Sous l'égide des gouvernements modérés, elles avaient concerné des surfaces réduites et un nombre limité de bénéficiaires; certaines avaient même été annulées ${ }^{7}$. Le projet politique de Tejeda visait à mettre en place un régime socialiste laissant une large autonomie aux municipalités, dont la coordination avec le pouvoir provincial était assurée par une organisation paysanne unifiée, la Ligue des Communautés Agraires de l'Etat de Veracruz (LCAEV) ${ }^{8}$. Sous son mandat, les conseils municipaux se virent allouer de nouveaux moyens juridiques - la capacité de former des Juntes d'arbitrage en matière foncière et d'imposer aux propriétaires des contrats de fermage pour cause d'utilité

\footnotetext{
${ }^{5}$ Pour une présentation détaillée du processu d'éviction des grands commerçants de San Andrés et leur remplacement par une nouvelle classe d'entrepreneur liés au négoce international, voir Léonard, "Marché foncier, asymétries de pouvoir et exclusion...", op. cit.

${ }^{6}$ Alfredo Delagdo Calderón, “Espacios y luchas revolucionarias en el Sotavento veracruzano”, in E. Velázquez, E. Léonard, O. Hoffmann, M.-F. Prévôt-Schapira (coords.), El Istmo mexicano: una región inasequible. Estado, poderes locales y dinámicas espaciales (siglos XVI-XXI), CIESAS-IRD, México, 2009: 353-392.

7 Voir José Velasco Toro, Tierra y conflicto social en los pueblos del Papaloapan veracruzano (1521-1917), Xalapa, Universidad Veracruzana, 2003.

8 Voir Heather Fowler-Salamini, Movilización campesina en Veracruz (1920-1938), Mexico DF, Siglo XXI Editores, 1979 ; Eitan Ginzberg, "Formación de la infraestructura política para una reforma agraria radical: Adalberto Tejeda y la cuestión municipal en Veracruz, 1928-1932", Historia Mexicana, 2000, XLIX (4): 637-727.
} 
publique, de façon à permettre l'implantation de groupes de paysans alliés à la LCAEV qui se constitueraient ensuite en comités agraires - et militaires - via la formation et l'armement de milices à partir des comités agraires ${ }^{9}$. À travers ces instruments, Tejeda ouvrait un espace à la restauration de pouvoirs municipaux en situation de fusionner les sphères foncière, politique et, dans une certaine mesure, militaire - une prérogative dont elles avaient disposé avec les Gardes Nationales durant le premier demi-siècle suivant l'indépendance.

En 1922, la municipalité contrôlée par le Parti Rouge formula une demande de restitution de terres en réparation des spoliations perpétrées à l'occasion du démantèlement du commun municipal. Mais cette demande fut accompagnée de sollicitudes similaires émanant de treize villages situés sur l'ancien "commun". La formulation conjointe de revendications concurrentes suggère que les autorités des villages concernés, ou pour le moins des secteurs de leur population, se défiaient de la reconstitution d'une propriété communale sous l'égide du gouvernement municipal, à l'instar de la situation qui avait prévalu jusqu'au milieu des années 1880.

Les instances supérieures d'arbitrage manifestèrent une divergence de position similaire. Alors que, dans une résolution juridique de mai 1923, le gouverneur Tejeda avait considéré que « la pétition de San Andrés Tuxtla, ainsi que celles de toutes les congrégations qui constituent le Municipe du même nom doivent être résolues dans le même dossier, afin d'éviter [...] que des décisions contradictoires se produisent s'agissant d'une même restitution ${ }^{10}$, le président de la République Calles pris l'exact contre-pied de cette sentence, en révoquant trois ans plus tard la résolution de restitution, déniant à la ville de San Andrés toute compétence juridique pour recevoir des terres, et en allouant à chaque village une dotation agraire séparée ${ }^{11}$. Cette décision conduisait à fractionner les instances légales de gouvernement foncier et mettait en relief le souci de l'exécutif fédéral d'éviter la consolidation dans le Veracruz d'un complexe politique en situation de défier son pouvoir, autour de l'alliance entre la LCAEV, le gouvernement provincial et les municipalités.

Quoi qu'il en fut, dans un contexte de résurgence de la violence entre milices paysannes, guardias blancas des grands propriétaires et éléments de l'armée favorables aux intérêts de ces derniers, la municipalité joua un rôle central de pilotage des processus d'allocation et de gestion des terres. A travers la tutelle de la LCAEV sur les milices paysannes et les liens qui existaient entre les dirigeants des comités agraires locaux et ceux du municipe, la municipalité a opéré comme instance centrale dans l'identification des bénéficiaires de terre, en liant notamment l'exercice des droits fonciers à l'allégeance vis-à-vis de la Ligue et aux mérites militaires acquis dans les conflits engageant cette dernière contre les groupes opposants.

\section{Fracture du mouvement agrariste et conflits autour des terres redistribuées}

A partir de 1927, toutefois, la mainmise du gouvernement municipal sur la politique foncière locale a été l'objet de différentes remises en question. La première fracture, de nature politique, était interne au mouvement agrariste et avait pour enjeu le contrôle du gouvernement municipal. Elle opposait les deux figures principales de la Ligue, Manuel Azamar, un dirigeant paysan qui avait fondé sa stratégie sur le contrôle des instances locales de gouvernement politique et foncier, et Primitivo Valencia, un officier de l'armée révolutionnaire qui disposait de relations solides au sein de l'appareil militaire et politique provincial. On peut analyser leur opposition en termes de confrontation entre deux projets divergents de construction politique : le premier, de type autonomiste, était centré sur le contrôle des dispositifs locaux d'accès aux ressources naturelles à partir de la municipalité ; celui de Valencia reposait sur l'incorporation fonctionnelle du mouvement agrariste tuxtlèque à des réseaux politiques de portée nationale.

\footnotetext{
9 Romana Falcón \& Soledad García. La semilla en el surco. Adalberto Tejeda y el radicalismo en Veracruz (1883-1960), Mexico DF, El Colegio de México-Gobierno del Estado de Veracruz, 1986: 127 et ss.

${ }^{10}$ Gaceta Oficial del Gobierno del Estado de Veracruz, no 131, du 2 novembre 1926: 8.

${ }^{11} \mathrm{Ibid}$. Ces dotations multiples à un total de 16 localités portaient sur $10300 \mathrm{Ha}$, alloués à 1961 paysans.
} 
L'éclatement de l'antagonisme entre ces deux figures a coïncidé avec le développement de tensions qui s'exprimaient dans deux sphères de relations politico-territoriales. La première concernait les rapports entre les localités bénéficiaires d'une dotation agraire et le centre du pouvoir municipal. La seconde était interne aux ejidos et opposait certains comités agraires locaux et des hameaux sujets à leur autorité. Ces tensions avaient pour objet commun la régulation des droits d'usage des ressources (terres, pâturages, zones boisées) et la légitimité des autorités impliquées dans cette régulation. Elles sont révélées par une série de plaintes formulées par des comités locaux au sujet des intromissions de la municipalité ou du Comité agraire municipal présidé par M. Azamar dans la distribution des droits d'usage locaux ${ }^{12}$.

Ces plaintes étaient de deux ordres : certaines dénonçaient les accaparements de ressources réalisés par des chefs de milice ou par le Comité directeur de la Ligue agraire tuxtlèque, présidé par Azamar, pour louer les terres redistribuées ou attribuer des permis d'exploitation forestière à des membres de l'ancienne élite agraire et commerciale ; une autre série de dénonciations émanait de hameaux qui avaient bénéficié d'une dotation foncière sous la tutelle d'un village de taille plus importante et qui réclamaient leur ségrégation administrative et leur indépendance dans la gestion des ressources naturelles ${ }^{13}$. Ces plaintes présentaient de fortes similitudes avec celles qui avaient été dirigées contre la Société Agricole de San Andrés au cours des années 1870 et 1880.

Ces tensions furent momentanément résolues à l'occasion d'un nouveau pronunciamiento militaire, à l'approche des élections présidentielles de 1929, lors duquel Valencia et un certain nombre des comités agraire de la région prirent le parti des insurgés. En mars, Valencia fut arrêté et exécuté et un mois plus tard, Azamar informait la Commission Agraire de l'Etat de Veracruz " que les comités [locaux auteurs de plaintes à son encontre] ont été destitués pour avoir pris les armes [...] et un nouveau comité a été nommé ${ }^{14}$. Ce dénouement semblait sceller le triomphe du projet autonomiste et la consolidation de la "municipalité agraire". En fait, l'avenir de ce projet était lié à celui du gouverneur Tejeda et de sa stratégie de conquête du pouvoir national. Lors de l'élection présidentielle de 1934, Tejeda fut défait par le candidat du parti officiel, Lázaro Cárdenas, qui prônait lui aussi une intensification de la réforme agraire. Mais, à la différence de Tejeda, Cárdenas visait à utiliser les redistributions de terre comme levier pour réduire l'autonomie des communautés rurales et forcer leur incorporation à l'Etat.

\section{La réforme agraire comme processus de frontière : ruptures intergénérationnelles, segmentation sociale et recomposition des structures politico-territoriales}

\section{La mise sous tutelle de la municipalité et l'implantation d'un nouveau dispositif d'intervention étatique}

Sous la présidence de Cárdenas (1934-1940), la politique de réforme agraire a radicalement changé d'intensité et d'orientation. Cette impulsion a été permise par la crise mondiale des années trente et l'affaiblissement concomitant des coalitions régionales qui restaient marquées par l'influence des élites exportatrices agricoles et minières. La grande crise a créé les conditions d'une inversion du rapport de forces entre celles-ci et l'Etat national. La réforme agraire est alors devenue l'instrument central d'un projet de refondation du régime de gouvernementalité rurale s'appuyant sur une transformation des structures politico-territoriales.

Alors que les dotations foncières avaient jusqu'alors été limitées aux ressortissants des communautés spoliées de leurs terres et à des surfaces réduites, de faible qualité agricole, la réforme du Code agraire de 1934 en ouvre l'accessibilité aux employés et tenanciers des haciendas et même les plus

\footnotetext{
12 Entre 1930 et 1932, Azamar cumula les fonctions de maire et de président du Comité agraire municipal.

${ }^{13}$ Pour un panorama détaillé des ces conflits locaux, voir les cas présentés par Léonard, Dynamique du changement légal..., op. cit.: 176-178.

${ }^{14}$ Archivo de la Comisión Agraria Mixta de Veracruz, Exp. 328, 1929.
} 
productives de celles-ci deviennent des sujets d'expropriation ${ }^{15}$. Entre 1935 et 1940, les dotations de terre concernent une surface double de celle qui avait été affectée jusqu'alors ${ }^{16}$. On assiste surtout à une reprise en main de la politique agraire par l'Etat, qui contourne les institutions sociopolitiques existantes, communautés et municipalités, au profit d'une relation directe entre l'administration fédérale et des groupes paysans dont les représentants locaux de l'Etat organisent la constitution et la reconnaissance légale. La réforme agraire opère dès lors comme un instrument d'incorporation des communautés rurales à un projet unifié d'Etat-Nation, planifié et mis en œuvre de façon centralisée. L'une de ses dimensions les plus significatives a consisté dans la formation de communautés plus nombreuses, mais de taille réduite, dont la fragmentation correspondait à un démantèlement des organisations socio-territoriales, de type communal ou latifundiaire, qui préexistaient à la formation de l'Etat postrévolutionnaire.

Ce processus de recomposition des structures socio-territoriales et de marginalisation institutionnelle des instances municipales et provinciales se combine avec la mise en place d'un dispositif centralisé de médiations avec le secteur paysan. Celui-ci s'appuie sur deux piliers : d'une part l'administration agraire, qui traite les demandes de dotation de terres, et d'autre part, une organisation corporative unifiée, elle même instituée en pilier du parti officiel, à laquelle les différents syndicats paysans sont enjoints de s'incorporer et qui reçoit un semi-monopole de canalisation des demandes paysannes et de désignation des représentants politiques de ce secteur ${ }^{17}$.

Dès le début de l'année 1935, le dispositif étatique s'étoffe, avec l'implantation d'une délégation régionale du Département Agraire à San Andrés et l'arrivée d'activistes agraristes venus d'autres régions. Au cours de la décennie suivante, toutes les élections municipales sont invalidées et les maires sont désignés par le gouverneur provincial, le plus souvent parmi un personnel étranger à la région ou qui s'en est éloigné de longue date. Au printemps 1935, M. Azamar, qui exerçait la primatie indiscutée sur le mouvement paysan régional, est nommé à un poste de haut fonctionnaire à Mexico, où il résidera jusqu'en 1956. Cet exil décapite la Ligue des communautés agraires et le Parti Rouge et inaugure une période de luttes internes, que met à profit l'administration fédérale pour rendre impossible toute reconstitution d'un front unifié autour de la municipalité.

\section{L'activation d'une frontière interne : ruptures intergénérationnelles et fragmentation territoriale}

Ces transformations des champs institutionnel et politique coïncident avec un bouleversement des rapports internes aux sociétés villageoises. À partir de 1934, les tensions qui s'étaient faites jour à la fin de la décennie précédente entre certains villages et le centre municipal, ou entre des chefs-lieux d'ejido et des hameaux-sujets, connaissent un regain d'expression. On assiste à l'insurrection de groupes sociaux situés en position subalterne dans les chaînes d'autorité locale, comme dans les ejidos de Soyata, Comoapan, Tulapan, Xoteapan et Chuniapan ${ }^{18}$. Ces groupes revendiquent leur séparation de leur communauté de tutelle et la formation d'un ejido indépendant, soit à travers une nouvelle dotation foncière, soit par ségrégation des terres qu'ils occupent et l'élection de leurs propres autorités. Les occupations de terres se multiplient, sur les grandes propriétés situées dans l'aire centrale de la région, mais surtout sur le pourtour du territoire municipal, là où la réforme agraire n'avait pas eu d'incidence. Le plus souvent, la junte municipale nommée par le gouverneur

\footnotetext{
${ }^{15}$ Horacio Mackinlay, "La política de reparto agrario en México (1917-1990) y las reformas al artículo 27 constitucional”, in A. Massolo, M. Bassols (coords), Procesos rurales y urbanos en el México actual, Mexico, UAM, 1991: 117-167.

${ }^{16} 20,1$ millions d'hectares entre 1935 et 1940 , contre 11 au cours des 20 années précédentes ; le nombre des ejidos a été multiplié par cinq et celui des ménages bénéficiaires s'est accru de 82\% seulement. Voir Mackinlay, op. cit.: 133-134.

${ }^{17}$ Voir Will Pansters, "Theorizing Political Culture in Modern Mexico", in W. Pansters (ed.), Citizens of the Pyramid. Essays on Mexican Political Culture, Amsterdam, Thela Publishers, 1997: 1-37.

${ }^{18}$ Voir Eric Léonard, “La réforme agraire comme processus de frontière. Logiques d'autonomisation, ancrage de l'État et production institutionnelle dans la région des Tuxtlas (Mexique)", Autrepart, 30, 2004: 97-116.
} 
régularise ces invasions par le biais de contrats de fermage obligatoire, qui permettent la formation de nouveaux hameaux habilités à formuler des demandes de dotation foncière.

Ces dynamiques correspondent à une recomposition spectaculaire des structures territoriales et démographiques. Alors que 16 ejidos avaient été créés entre 1923 et 1933, la décennie suivante voit se former 32 nouvelles communautés (figure 1). Le nombre de bénéficiaires passe de 1960 à 4 617, et la superficie redistribuée est multipliée par 3,4. Mais au rebours de l'iconographie officielle, faisant des ouvriers et tenanciers des domaines expropriés les grands bénéficiaires des distributions de terre, les adhérents des nouveaux ejidos viennent dans leur majorité de villages éloignés des haciendas concernées, alors que les employés de ces dernières se plaignent à l'administration d'être victimes de discriminations ou de menaces d'expulsion de la part des comités conduisant les démarches de dotation.

En fait, le territoire municipal est le théâtre d'un gigantesque brassage de population entre 1930 et 1940. En premier lieu, les villages qui avaient bénéficié de dotation agraire dans les années vingt se vident : la population de Xoteapan, par exemple, passe de 1069 à 415 habitants (-61,5\%), celle de Ranchoapan de 764 à $268(-65 \%)$, celle de Tulapan de 585 à $341(-42 \%)^{19}$. Le centre de peuplement historique du municipe perd au total $10 \%$ de sa population au cours des années trente. En revanche, les zones périphériques voient leur population tripler. Cet essor bénéficie en partie aux hameaux de travailleurs des haciendas situées dans la partie Sud du municipe, mais il correspond principalement à la formation de nouvelles localités : entre 1930 et 1940, 22 nouveaux villages sont créés, soit un accroissement de $75 \%$ à l'échelle du municipe. Ces nouveaux centres de peuplement absorbent au total $85 \%$ du croît démographique dans les zones périphériques.

Bien plus qu'un simple transfert de population, la réforme agraire induit une véritable recomposition des rapports sociaux internes aux communautés villageoises. Les caractéristiques de la population bénéficiaire des nouvelles dotations de terre révèlent en effet une rupture intergénérationnelle à l'échelle des anciens villages du commun municipal. Alors que durant les années 1920, ces dotations ont bénéficié en majorité à des hommes de plus de 35 ans, dix ans plus tard elles concernent en priorité des jeunes de 15 à 24 ans, qui représentent $40 \%$ des nouveaux ejidatarios, contre à peine plus de $20 \%$ auparavant. La réforme agraire apparait soutenir les stratégies d'émancipation des jeunes adultes vis-à-vis des structures patriarcales de leur localité d'origine. A Cuesta Amarilla, le comité agraire de 5 membres, compte trois individus de moins de 20 ans, quand un seul en a plus de $25^{20}$. Parfois, à peine formés, les ejidos sont confrontés aux tentatives de sécession d'une partie de leurs adhérents : créé en 1935, lors de la fondation de l'ejido Mata de Caña, le hameau de La Boca constitue son propre comité agraire deux ans plus tard et sollicite l'érection d'un ejido indépendant $^{21}$. En 1942, Soyata vit une expérience similaire, lorsqu'un groupe de 15 de ses jeunes ressortissants s'installe sur une portion périphérique de son terroir, au lieu-dit La Redonda, et recrute des adhérents dans les ejidos voisins pour entamer une procédure de ségrégation.

\footnotetext{
${ }^{19}$ Dirección General de Estadísticas, V Censo de Población, 1930, Mexico, 1934 ; VI Censo de Población, 1940, Mexico, 1943.

${ }^{20}$ Archivo del Registro Agrario nacional, San Andrés Tuxtla, Exp. 2850, 1942.

${ }^{21}$ Archivo de la Comisión Agraria Mixta de Veracruz, exp. 739, dotación, 1937.
} 


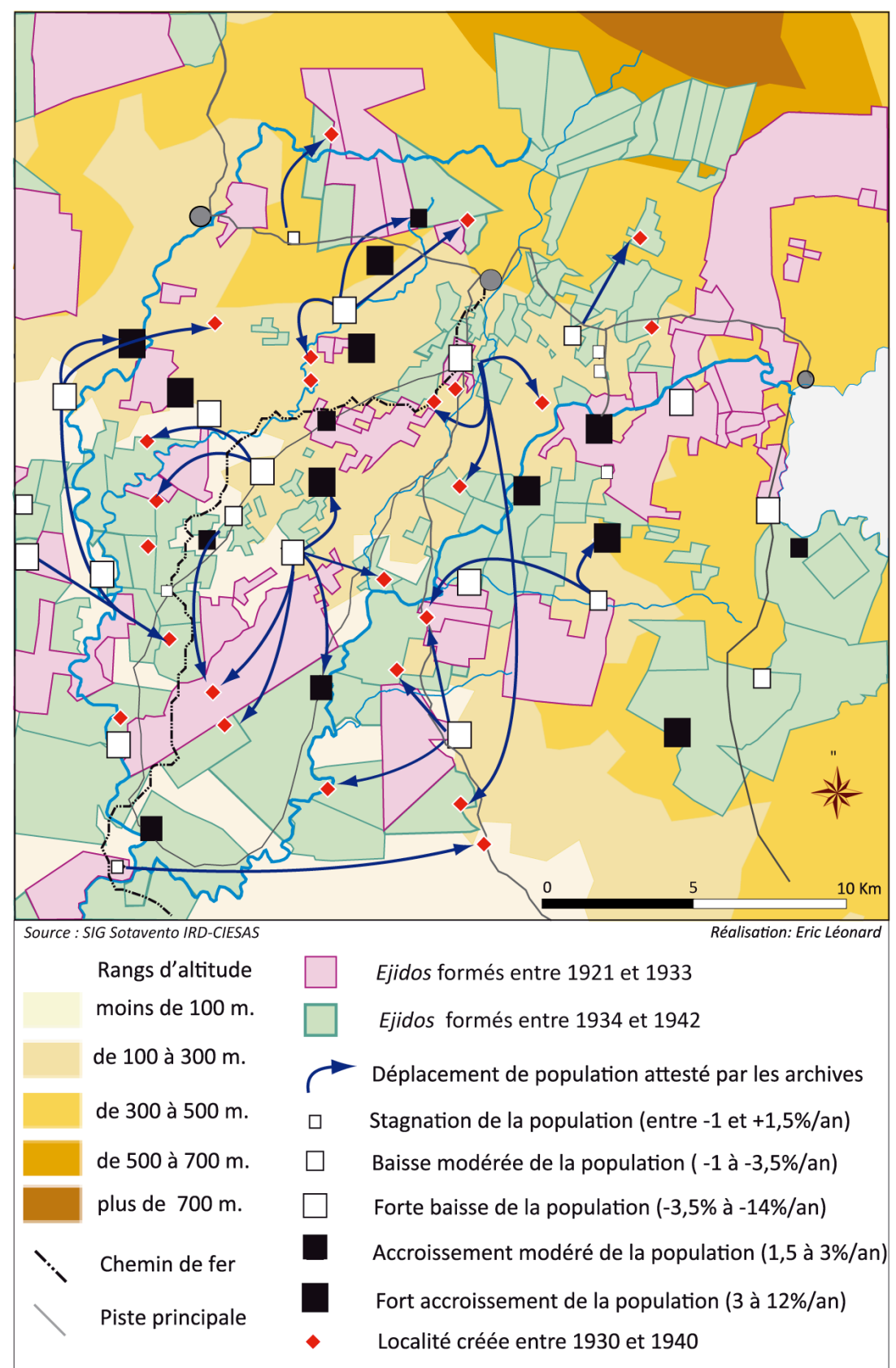

Sources : Recensements du Département Agraire ; Recensements de population 1930 et 1940

Ces dynamiques de redistribution du peuplement et d'émancipation ne sont spécifiques ni à la région, ni à la période de la réforme agraire. L. Cambrézy constate la coïncidence entre l'activation à grande échelle de la réforme agraire et la formation des localités qui forment aujourd'hui la trame territoriale du centre de l'Etat de Veracruz ${ }^{22}$. Mais plus généralement, cette situation s'inscrit dans les logiques historiques de segmentation sociale et de mobilité ayant travaillé les sociétés indiennes dans les périodes de changement institutionnel depuis l'époque coloniale ${ }^{23}$.

\footnotetext{
${ }^{22}$ Luc Cambrézy, "Terre et territoire au Mexique (Veracruz). De la réforme agraire à la fiction municipale", Cahiers des Sciences Humaines, 28 (4), 1992: 625-642.

${ }^{23}$ Voir Jonathan D. Amith, The Möbius Strip. A Spatial History of Colonial Society in Guerrero, Mexico, Stanford, Stanford University Press, 2005.
} 
Au centre de ces processus, on retrouve une figure elle aussi transversale de l'histoire des sociétés indiennes : celle du jeune entrepreneur politique qui, à travers la mobilité spatiale, la valeur militaire et/ou l'établissement de liens avec les représentants de l'Etat cherche à contourner les structures patriarcales d'autorité de sa société d'origine et accéder à des positions de pouvoir ${ }^{24}$. La politique de réforme agraire fournit de nouvelles ressources institutionnelles et politiques à l'expression de telles stratégies. Les dirigeants des nouveaux ejidos sont en majorité de jeunes villageois qui ont tissé des liens personnels au sein du dispositif local de l'Etat (Département Agraire, armée, organisations corporatives officielles) ${ }^{25}$. Ils s'appuient sur la stratégie étatique de marginalisation des organisations communales pour contourner les structures de pouvoir des anciens villages et se positionner sur les frontières territoriales, là où le processus de réforme agraire contribue à créer des espaces institutionnels purgés des systèmes de droits et d'autorités antérieurs. Ils y entreprennent de construire des monopoles de courtage économique et politique qui leur permettent d'organiser les territoires émergents en marchés captifs.

Car, en brisant les structures de contrôle productif et marchand qui opéraient au sein des grands domaines, la réforme agraire impulse une expansion spectaculaire de la production paysanne de grains (maïs, haricot, riz), de saindoux et de cassonade. Cette ouverture des marchés locaux permet le développement d'un secteur commercial qui avait jusqu'alors végété à l'ombre du système latifundiaire. Avec l'appui de sociétés industrielles du centre du pays, un groupe de commerçants entreprend de subventionner l'activité des comités agraires sur la frontière et de tisser avec eux un dispositif personnalisé de financement et de contrôle des récoltes. Au cours des années 1930 et 1940 se noue ainsi une imbrication d'intérêts entre quelques grands commerçants de grains et le dispositif de gouvernance de la réforme agraire (agents du Département Agraire, délégués des syndicats, dirigeants d'ejidos), dont l'établissement de relations d'exclusivité - commerciale et politique - avec les ejidos est l'enjeu.

Les ressources sur lesquelles les entrepreneurs de frontière peuvent asseoir leur démarche de construction et de contrôle de nouveaux territoires sont ainsi de deux ordres : d'une part, les réseaux de patronage politique et économique dont San Andrés est le centre de coordination et, d'autre part, les groupes sociaux subalternes dans les hiérarchies villageoises au sein desquels ils recrutent leurs adhérents. Leur crédibilité et leur autorité reposent sur les garanties qu'ils peuvent offrir à leur clientèle en termes d'accès aux dotations foncières et aux crédits à la production. Réciproquement, ils doivent fournir à leurs réseaux de patronage politique et commercial des garanties quant à la taille et à la loyauté de leurs clientèles locales. Ce double jeu de contraintes conduit les entrepreneurs de la frontière à investir une fonction "passeurs institutionnels" entre deux registres de règles: $d$ 'une part, celles qui organisent le fonctionnement du dispositif local de l'Etat, autour du cadre légal de la réforme agraire et de l'institutionnalisation des médiations corporatives; et d'autre part les normes sociales qui organisent les échanges au sein de la société villageoise, sous la grammaire de l'échange réciproque et de l'économie morale communautaire ${ }^{26}$. Ce second registre de légitimation conduit à ouvrir l'accès aux droits fonciers, que le cadre légal restreint aux individus enregistrés par l'administration étatique, à l'ensemble des chefs de familles, indépendamment de leur statut

\footnotetext{
${ }^{24}$ Sur les stratégies de promotion sociale des jeunes indiens à travers le prestige militaire au cours du premier siècle d'indépendance au Mexique et au Pérou, voir Florencia Mallon, Peasant and Nation. The Making of Postcolonial Mexico and Peru, Berkeley, Los Angeles \& London, University of California Press, 1995.

${ }^{25}$ Jan Rus observe des dynamiques similaires dans les hautes terres du Chiapas à la même époque : J. Rus, "The 'Comunidad Revolucionaria Institucional': The subversion of Native Government in Highland Chiapas, 1936-1968", in G. M. Joseph, D. Nugent (eds), Everyday Forms of State Formation. Revolution and the Negotiation of the Rule in Modern Mexico, Durham \& London, Duke Univesity Press, 1994: 263-300.

${ }^{26}$ Voir James C. Scott, The moral economy of the peasant: Rebellion and subsistence in Southeast Asia, New Haven, Yale University Press, 1976.
} 
officie $^{27}$. L'intégration de l'ensemble des adultes résidents (ejidatarios comme nouveaux arrivants) en tant que producteurs autonomes participe ainsi de l'élargissement de la clientèle du dirigeant local et de la "grandeur" de sa communauté.

Ces situations de pluralisme normatif supportent l'institutionnalisation du patronage clientéliste en tant que principe de régulation sociopolitique. Elles légitiment le monopole exercé par l'entrepreneur de frontière sur les fonctions de médiation politique et commerciale et justifient son exercice autoritaire du pouvoir, la privation de droit et l'expulsion des individus qui, par la contestation de sa légitimité, mettent en danger l'équilibre de la communauté paysanne, son existence même, dans un contexte de violence et d'insécurité liées à la concurrence exacerbée pour le contrôle des territoires de la frontière agraire.

Car les logiques de construction et d'extension des clientèles politiques et commerciales s'articulent aux tensions qui opposent les factions politico-syndicales en concurrence pour le contrôle du mouvement agrariste régional, elles-mêmes médiatisées par les jeux d'alliance et de confrontation entre les centrales corporatives qui luttent pour la représentation paysanne au niveau national. Dans les Tuxtlas, ces luttes mettent aux prises trois fédérations : la Ligue des Communautés Agraires, qui conserve une base importante dans le centre de la région; la Confédération des Travailleurs Mexicains (CTM), qui bénéficie d'un soutien fort de l'administration fédérale, notamment à travers l'armement des "défenses rurales" qui protègent les comités agraires; et la Confédération Ouvrière et Paysanne du Mexique (COCM), dont l'audience s'accroît à la fin des années 1930, à partir de ses bases d'implantation originelle dans les zones sucrières au sud de la région.

A partir de 1938, les processus de formation de nouveaux ejidos sont enchâssés dans les logiques d'extension du contrôle territorial des dirigeants locaux de ces factions. Les expulsions violentes, les raids contre les installations commerciales, les incendies de cultures ou de récoltes, les assassinats de membres des comités agraires affiliés à l'un ou l'autre deviennent courantes. Cette violence a pour premier effet de relancer la mobilité impulsée par la politique de dotations agraires. En 1939, un an après la création de l'ejido Abrevadero, sous les auspices de Juan Paxtián et de la CTM, un groupe de bénéficiaires, parrainés par Nicolás Parra et la COCM, fait sécession et s'en va occuper une propriété voisine, au lieu-dit Sayultepec, où il fait une demande de dotation indépendante qui sera satisfaite quatre ans plus tard. À Tulapan, les dissidents du gouvernement local de Juan Atén (CTM) sont expulsés et leurs droits fonciers sont réalloués à des familles venues de Cuesta Amarilla, Los Naranjos et El Remolino, sur une base clientéliste (figure 2).

Ce n'est qu'après l'alternance présidentielle de 1941 que l'on assiste à une pacification progressive et une reprise en main par le dispositif étatique. À partir de 1943, les éléments les plus radicaux des différents camps sont cooptés ou mis au pas, de façon souvent brutale, par l'appareil officiel. Le pacte national de gouvernementalité corporative est tranché en faveur de la Confédération Nationale Paysanne (CNC), dont est membre la Ligue des Communautés Agraires. Durant l'année 1945, les derniers éléments de dissidence sont mis au pas: J. Paxtián est nommé à un poste administratif au siège de la CTM, à Mexico, alors que M. Parra et l'un de ses principaux lieutenants sont assassinés en mars et juillet de la même année ${ }^{28}$. Les figures radicales historiques de la LCA sont pour leur part marginalisées par l'ascension d'une nouvelle génération de dirigeants, aux ordres du pouvoir central et qui restera en place jusqu'au milieu des années 1970.

\footnotetext{
${ }^{27}$ Pour une description des systèmes normatifs locaux, voir E. Léonard, Dynamique du changement légal..., op. cit.: 216-218.

${ }^{28}$ Léon Medel, Historia de San Andrés Tuxtla, Xalapa, Gobierno del Estado de Veracruz, 1993, T. II: 540-542.
} 


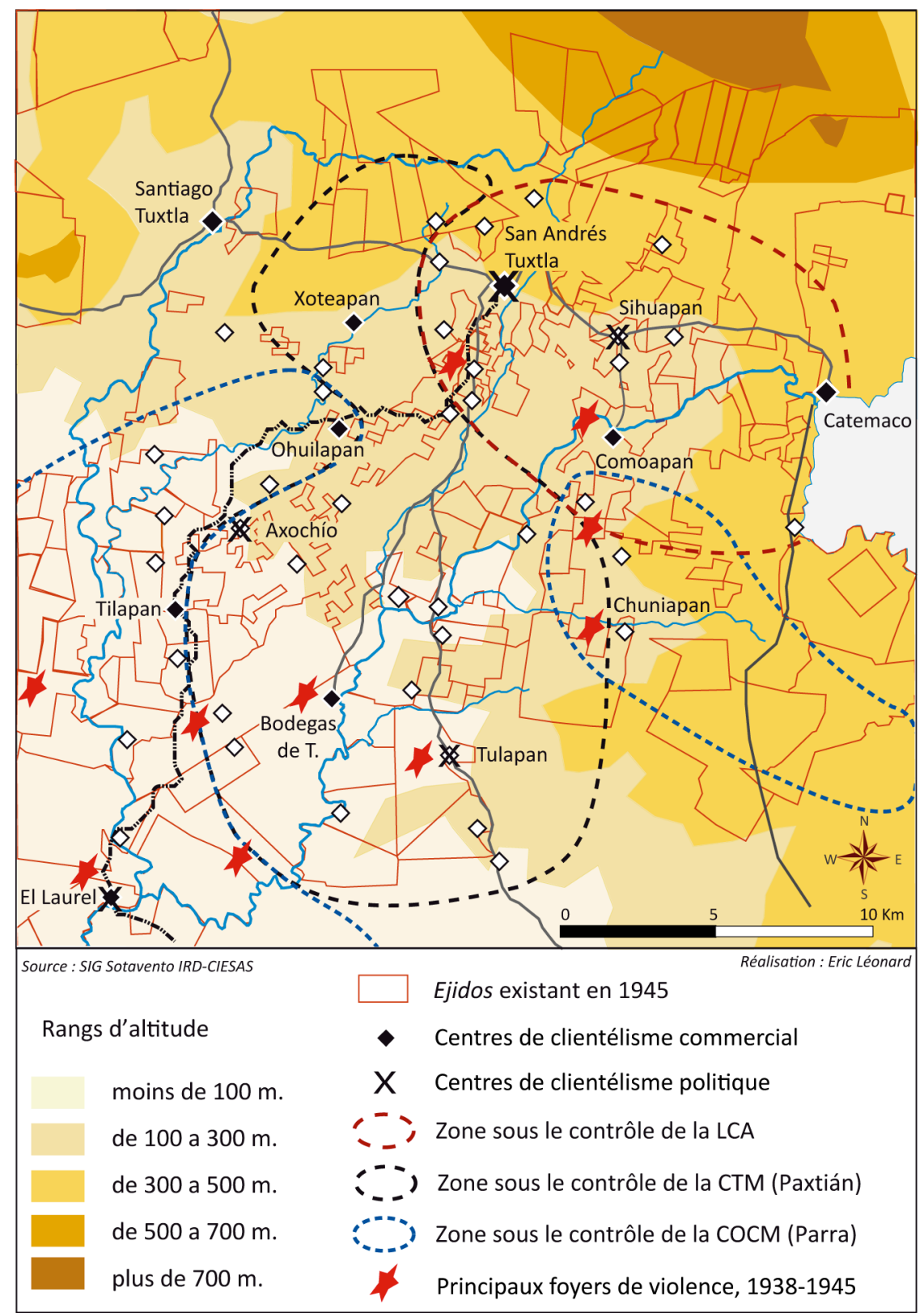

\section{Conclusion : dynamique de la frontière agraire, violence et construction du régime de gouvernementalité rurale}

Le mode de gouvernementalité, qui prend une forme stabilisée au milieu des années 1940, peut être caractérisé par la combinaison d'un pouvoir bureaucratique, incarné dans le dispositif local de l'Etat, et un pouvoir de type despotique à l'échelon local, qui actualise certains traits des pratiques de caciquat des époques coloniale et libérale, tout en étant soumis au contrôle du dispositif de l'Etatparti. Le mode de régulation de ce régime est constitué principalement par les ressources foncières que l'Etat est en situation de mobiliser et de distribuer et par les crédits à la production dont les grands commerçants de San Andrés sont les opérateurs principaux. La coordination de ce système est organisée dans une large mesure par l'appareil bureaucratique de l'Etat local: les agences de l'administration fédérale et le comité régional du parti officiel, au sein duquel figurent les représentants de la fédération unique des syndicats paysans. 
Les contraintes de clientélisation des organisations locales par l'appareil bureaucratique de l'Etatparti laissent toutefois des marges de manœuvre aux dirigeants locaux, tant qu'ils reconnaissent la prééminence des directions syndicales dans la négociation des compromis politiques. Les dirigeants des ejidos constituent le maillon essentiel de la chaîne verticale des médiations personnalisées qui lient les communautés rurales au pouvoir central. À ce niveau également, la relation de patronage et les valeurs de loyauté et de réciprocité opèrent comme le ciment de l'incorporation des ejidos au dispositif étatique ${ }^{29}$. Le procesus d'institutionalisation locale de l'Etat à travers la réforme agraire actualise ainsi les pratiques historiques de patronage personnalisé dans la construction des nouvelles organisations politiques rurales, qu'elles soient de nature corporative (syndicats) ou territoriale (ejidos). Ce faisant, il réaffirme les principes de personnalisation du pouvoir étatique dans les mains d'une poignée d'acteurs locaux, ainsi que le mode clientéliste de régulation de l'accès aux ressources. L'élément innovateur de ce processus ne réside pas dans la délégation personnalisée du pouvoir étatique ${ }^{30}$, mais dans l'institutionnalisation des instances de médiation incorporées à l'Etat, qui opèrent comme espaces où se négocient les formes d'assignation des ressources et s'affirme dans le même temps le caractère délégué de ces ressources.

Le type de gouvernementalité adopte ainsi des traits caractéristiques du "despotisme décentralisé" analysé par M. Mamdani en Afrique sub-saharienne ${ }^{31}$. Il correspond à un double enjeu d'ancrage dans les campagnes d'un dispositif étatique jusqu'alors concentré dans les grandes villes, et de stabilisation $d^{\prime} u n$ ordre politique négocié, de nature non démocratique et par nature instable ${ }^{32}$. Le processus de réforme agraire tuxtlèque peut être assimilé à un processus de colonisation interne par l'Etat de ses marges territoriales dans un contexte de forte autonomie des pouvoirs locaux et régionaux ${ }^{33}$. Dans ces contextes, les entrepreneurs de frontière ont incarné à la fois le pouvoir étatique et la légitimité politique communautaire, en remplissant une double fonction de vecteurs/traducteurs des normes d'incorporation politique et économique des communautés au système national et de portesparole/interprêtes exclusifs des expressions politiques de ces communautés.

Les traits essentiels du mode de gouvernementalité de la frontière agraire mexicaine (l'enracinement des pratiques de l'État local dans la culture politique des sociétés indiennes, le fondement de l'autorité sur le principe d'antériorité dans la construction des rapports avec la bureaucratie étatique et les commerçants, la grammaire de l'échange réciproque comme support des relations politiques) sont analogues aux principes de fonctionnement de la frontière interstitielle africaine ${ }^{34}$. Ils légitiment la détention viagère des charges d'autorité et la gestion patrimoniale des ressources territoriales par l'entrepreneur de frontière. Ils justifient également l'usage de la violence comme mode de régulation des tensions et dissensions politiques internes. Ce mode de gouvernementalité est indissociable de la dynamique renouvelée des dotations foncières, qui a permis de maintenir dans la durée l'accès des jeunes ruraux à la terre et aux ressources étatiques. L'épuisement de la frontière agraire, effectif depuis la fin des années 1970 et formalisé par la réforme constitutionnelle de 1992, a matérialisé l'impossibilité d'en reproduire les bases matérielles et les principes moraux de justification. II a conduit à ses remises en question violentes à travers les zones rurales à partir des années 1980 et avec une intensité accrue au cours de la décennie suivante.

\footnotetext{
${ }^{29}$ W. Pansters, op. cit.

${ }^{30}$ L'Etat postrévolutionnaire n'a fait qu'actualiser les formes de concentration des fonctions de gouvernement civil et de régulation commerciale qui opéraient aux époques coloniale, puis libérale. Voir à ce sujet Claudio Lomnitz, Modernidad indiana. Nueve ensayos sobre Nación y mediación en México, Mexico, Editorial Planeta, 1999, chap. 9.

${ }^{31}$ Mahmood Mamdani, Citizen and Subject. Contemporary Africa and the Legacy of Late Colonialism, Princeton, Princeton University Press, Princeton Studies in Culture/Power/History, 1996.

${ }^{32}$ Jean-Pierre Chauveau, "Introduction thématique : les jeunes ruraux à la croisée des chemins", Afrique Contemporaine, 214 (2), 2005: 22.

${ }^{33}$ Pour une analyse de même nature appliquée au cas français, voir Eugene Weber, La fin des terroirs. La modernisation de la France rurale, 1810-1914, Paris, Fayard-Éditions Recherches, 1983.

${ }^{34}$ Igor Kopytoff, "The internal african frontier: the making of african political culture", in I. Kopytoff (ed.), The African Frontier. The Reproduction of Traditional African Societies, Bloomington/Indianapolis, Indiana University Press, 1987: 3-84.
} 